\title{
Leukemia-related protein I6 (LRPI6) promotes tumor growth and metastasis in pancreatic cancer
}

This article was published in the following Dove Press journal:

OncoTargets and Therapy

\author{
Zheng Mo',* \\ Minggen $\mathrm{Hu}^{1, *}$ \\ Fei Yu ${ }^{2}$ \\ Lijuan Shao' \\ Kexing Fan ${ }^{1,3}$ \\ Shunchang Jiao'
}

'PLA General Hospital Cancer Center, PLA Postgraduate School of Medicine, Beijing, People's Republic of China; ${ }^{2}$ Department of Hepatobiliary Surgery, Beijing Tsinghua Changgung Hospital, Beijing, People's Republic of China; 'International Joint Cancer Institute, The Second Military Medical University, Shanghai, People's Republic of China

*These authors contributed equally to this work
Correspondence: Kexing Fan;

Shunchang Jiao

Chinese PLA General Hospital,

28 Fuxing Road, Haidian District,

Beijing 100853, China

Email kexingfan@I26.com;

jiaosc@vip.sina.com
Background: Leukemia related protein 16 (LRP16), one of the genes belonging to the macro domain family, has been found up-regulated in various tumors including testicles, ovaries and mucosa of colon and is associated with poor clinical outcomes.

Purpose: The objective of this study was to investigate expression pattern and biological roles of LRP16 in pancreatic cancer.

Patients and methods: Western blot and immunohistochemistry were used to investigate the expression of LRP16 in pancreatic cancer cell lines and tissues. qRT-PCR was utilized to examine LRP16 mRNA expression. Lentivirus based overexpression and knockdown of LRP16 was carried out in four pancreatic cancer cell lines (Panc1, CFPAC1, SW1990 and AsPC1). Cell proliferation, migration and invasion were determined by MTS and transwell assay, respectively. Flow cytometry was performed to investigate cell apoptosis. In vivo, the tumorigenic ability of LRP16 was determined in a NOD/SCID mouse model.

Results: In the present study, we found that LRP16 expression was increased in pancreatic tumor samples, compared with normal tissues. Moreover, the LRP16 expression was positive in $60.9 \%$ of 156 specimens and correlated with tumor size, clinical stage, distant metastasis and tumor differentiation. Multivariate Cox regression analysis revealed that the level of LRP16 expression was an independent prognostic factor for overall survival in pancreatic cancer patients. Furthermore, silencing of LRP16 significantly accelerated apoptosis, decrease proliferation, migration and invasion of pancreatic cancer cell lines in vitro. In contrast, overexpression of LRP16 attenuated apoptosis, promoted proliferation, migration and invasion. In addition, in vivo study revealed that down regulation of LRP16 could attenuate tumor growth and prolong the survival. On the contrary, up-regulation of LRP16 could promote tumor growth and shorten their survival.

Conclusion: These findings suggest that LRP16 played an oncogenic role in pancreatic carcinoma.

Keywords: leukemia-related protein 16, pancreatic cancer, proliferation, metastasis

\section{Introduction}

Pancreatic ductal adenocarcinoma (PDAC), which is known as pancreatic cancer, remains one of the most devastating cancers worldwide with a 5-year survival rate of $1.2 \%-6 \%$. It is usually diagnosed at the advanced stages, responds poorly to the available chemotherapeutics, and constitutes the major factor for high mortality rate. ${ }^{1-3}$ Promising new therapeutic options for this disease have been shown over the past years, but only limited clinical success was achieved. ${ }^{4}$ Therefore, there is still an urgent need for identifying new target molecules crucially affecting tumorigenesis, invasion, and prognosis in developing effective treatments for pancreatic carcinoma (PC).

Leukemia-related protein 16 (LRP16), localized on chromosome 11q12.1, belongs to the macro domain family and was initially shown to be associated with leukemia 
relapse. ${ }^{5}$ Previous studies reveal that the expression of LRP16 is related to tumor growth, metastatic potential, and prognosis in many malignancies such as breast cancer, gastric carcinoma, colorectal carcinoma, neuroendocrine lung tumors, and aggressive hematologic malignancies. ${ }^{6-10}$ However, the role and significance of LRP16 expression have not been explored in PC.

In this study, we examined LRP16 expression level in PC tissues and the relationship between LRP16 and clinical features and overall survival (OS) in PC patients. Furthermore, we investigated the effects of LRP16 knockdown and overexpression on tumorigenic ability and in PC cells. In vivo, the tumorigenic ability of LRP16 was determined in a Non-obese Diabetic/Severe Combined Immune-deficiency (NOD/SCID) mouse model. All these findings for the first time proved that LRP16 promoted tumor progression and metastasis in PC and that LRP16 expression has potential as an index for the prognosis of PC patients.

\section{Materials and methods}

\section{Tissue specimens and patients}

Formalin-fixed paraffin-embedded specimens were collected from 156 patients who were preliminarily diagnosed and confirmed histologically as having PC and underwent surgery between 2005 and 2010 at the Chinese People's Liberation Army General Hospital, Beijing, China. None of the patients had undergone preoperative radiotherapy or chemotherapy. Histopathological diagnosis was carried out by two pathologists. The detailed characteristics are depicted in Table 1. The follow-up duration of patients ranged from 6 to 60 months. OS was defined as the survival time after diagnosis. In addition, 16 specimens (including tumors and peritumoral tissues) of PC were collected within 30 minutes after surgical resection from 2014 to 2016. After resection, the tissues were put into liquid nitrogen and frozen immediately and stored at $-80^{\circ} \mathrm{C}$ for Western blot and RT-PCR analysis. The study was conducted after obtaining Institutional Ethical Committee approval from the PLA General Hospital and informed written consent from all of the patients.

\section{Cell lines and mice}

The PC cell lines Panc1, CFPAC1, Bxpc3, SW1990, AsPC1, and HPDE6-C7 were purchased from China Infrastructure of Cell Line Resources (Beijing, China) and cultured in Dulbecco's Modified Eagle's Medium (Thermo Fisher Scientific, Cleveland, OH, USA) supplemented with $10 \%$
Table I The relationship between LRPI6 expression and clinical significance

\begin{tabular}{|c|c|c|c|c|}
\hline \multirow[t]{2}{*}{ Characteristics } & \multirow[t]{2}{*}{$\mathbf{n}$} & \multicolumn{2}{|c|}{ LRP I 6 expression } & \multirow[t]{2}{*}{$P$-value } \\
\hline & & $\begin{array}{l}\text { Positive } \\
(n=95)\end{array}$ & $\begin{array}{l}\text { Negative } \\
(n=6 I)\end{array}$ & \\
\hline Age (years) & & & & 0.3 \\
\hline$<65$ & 103 & 66 & 37 & \\
\hline$\geq 65$ & 53 & 29 & 24 & \\
\hline Gender & & & & 0.04 \\
\hline Male & 102 & 56 & 46 & \\
\hline Female & 54 & 39 & 15 & \\
\hline Tumor size $(\mathrm{cm})$ & & & & $<0.01$ \\
\hline$\leq 4$ & 91 & 68 & 23 & \\
\hline$>4$ & 65 & 27 & 38 & \\
\hline Tumor location & & & & $<0.01$ \\
\hline Head & 86 & 65 & 21 & \\
\hline Body & 70 & 30 & 40 & \\
\hline Perineural invasion & & & & 0.068 \\
\hline Absent & 67 & 35 & 32 & \\
\hline Present & 89 & 60 & 29 & \\
\hline Clinical stage & & & & $<0.01$ \\
\hline Early stage & 52 & 13 & 39 & \\
\hline Advanced stage & 104 & 82 & 22 & \\
\hline Distant metastasis & & & & $<0.01$ \\
\hline No & 94 & 46 & 48 & \\
\hline Yes & 62 & 49 & 13 & \\
\hline Differentiation & & & & $<0.01$ \\
\hline Well & 24 & 8 & 16 & \\
\hline Moderate & 73 & 37 & 36 & \\
\hline Poor & 59 & 50 & 9 & \\
\hline
\end{tabular}

fetal bovine serum at $37^{\circ} \mathrm{C}$ in an atmosphere containing $5 \%$ $\mathrm{CO}_{2}$. BALB/c male nude mice were purchased from Vital River Laboratory Animal Technology Co., Ltd. (Beijing, China). The mice experiments were approved by the Animal Experimental Ethics Committee of PLA General Hospital and conducted in the animal facility of PLA General Hospital according to the animal experimental ethics committee guidelines of PLA General Hospital.

\section{Plasmid construction and cell transfection}

V5-tagged LRP16 was constructed by cloning the fulllength cDNA into PLVX-IRES-Puro vectors, yielding LV-LRP16. The negative control, called LV-Control, was empty vector with no cDNA fragment. We designed and synthesized three pairs of cDNA oligonucleotides to target LRP16 mRNA expression and performed BLAST searches using the National Center for Biotechnology Information expressed sequence tag database to ensure that the small hairpin RNA (shRNA) construct only targeted human LRP16 expression. 


\section{3-(4,5-Dimethyl-thiazol-2yl)-5-(3- carboxymethoxyphenyl)-2-(4-sulfophenyl)- $2 \mathrm{H}$-tetrazolium (MTS) assay}

Cell growth was measured by MTS assay (Promega Corporation, Fitchburg, WI, USA) in 96-well plates (1,000 cells/well) following the instructions of the manufacturer.

\section{Transwell migration and invasion assay}

The capacities for cell migration and invasion were analyzed by the transwell assay, which was carried out using transwell inserts ( $8 \mu \mathrm{m}$ pore size; Corning Incorporated, Corning, NY, USA) according to the manufacturer's protocol. For the migration assay, cells resuspended in serum-free medium were placed in the upper chamber without Martigel coating. For the invasion assay, cells were placed in the upper chamber, which was coated with Matrigel (BD Biosciences, San Jose, CA, USA). Then, the chambers were incubated in culture medium with $10 \% \mathrm{FBS}$ at $37^{\circ} \mathrm{C}$ in the lower chamber. After 24 hours, cells that did not migrate or invade were wiped out while the migrated and invaded cells were stained with $0.05 \%$ crystal violet for 30 minutes. Pictures were captured from five random fields under Olympus CKX41 inverted/phasecontrast microscope (Olympus Corporation, Toyko, Japan). Each experiment was repeated at least three times.

\section{Flow cytometry}

A total of $1 \times 10^{6}$ cells in PBS were stained with FITCAnnexin V and PI (BD Biosciences) in accordance with the manufacturer's instructions. Then, the apoptotic analysis of the samples was measured using flow cytometry. Data acquisition and analysis were performed on an FC $500 \mathrm{MPL}$ System (Beckman Coulter, Inc., CA, USA).

\section{Immunohistochemistry}

The tissue specimens were fixed in $10 \%$ buffered formalin. The tissues were cut into $5 \mathrm{~mm}$ slices and then embedded in paraffin for immunohistochemical examination. The detailed procedures for immunohistochemistry and evaluation of score are described elsewhere. ${ }^{7}$ The patients' medical records and histopathology of each specimen were reviewed.

\section{RNA extraction and $\mathrm{qPCR}$}

Total RNA was extracted using the TRIzol reagent (Qiagen NV, Venlo, the Netherlands), and the RNA samples were treated with DNase (Promega Corporation, Fitchburg, WI, USA). Reverse transcription was performed using a HighCapacity cDNA Reverse Transcription Kit (TaKaRa, Shuzo
Co., Shiga, Japan) according to the manufacturer's specification. Target gene-expression levels were quantified by real-time primer pairs using the SYBR Green Master Mix (TaKaRa). Quantitative RT-PCR was performed with the ABI 7500 system (Applied Biosystems, Darmstadt, Germany). The house-keeping gene GAPDH was included as an internal standard.

\section{Western blot analysis}

Harvested cultured cells were lysed in RIPA lysis buffer with proteinase inhibitor. Total cellular proteins were separated by SDS-PAGE and then transferred to PVDF membranes. After probing with antibodies, the antigen-antibody complex was visualized by Immobilon ${ }^{\mathrm{TM}}$ Western Chemiluminescence HRP Substrate (EMD Millipore, Billerica, MA, USA). The antibody against LRP16 was a goat polyclonal antibody from Santa Cruz Biotechnology, Inc. (Dallas, TX, USA).

\section{Statistical analysis}

All the data are expressed as the mean \pm SD. The associations between LRP16 expression and the clinical features of PC patients were analyzed using $\chi^{2}$ test. The survival rates were calculated using the Kaplan-Meier method, and the outcomes were evaluated with log-rank test. The univariate and multivariate Cox regression model analyses were used to analyze the prognostic factors. SPSS 19.0 software (IBM Corporation, Armonk, NY, USA) was used for the statistical analyses. $P<0.05$ was considered statistically significant.

\section{Results \\ LRPI6 expression in pancreatic tumor samples and cell lines}

To investigate the role of LRP16 in PC, we compared LRP16 expression levels in 16 case-matched tumor and normal tissues from surgical specimens of PC patients using RT-PCR and Western blot analysis. LRP16 expression was elevated in tumor tissues compared with normal tissues (Figure 1A and B). Then, we tested how LRP16 was expressed in cultured PC cell lines and normal pancreatic cell line HPDE6-C7. We conducted Western blot analysis and qRT-PCR on five PC cells and HPDE6-C7. These results showed that LRP16 was substantially increased in PC cell lines compared to that of the HPDE6-C7 cell line (Figure 1C and D). Next, we evaluated immunohistochemically the expression of LRP16 in PC samples and adjacent nontumor tissues. IHC analysis showed that LRP16 expression was positive in tumor samples 
A

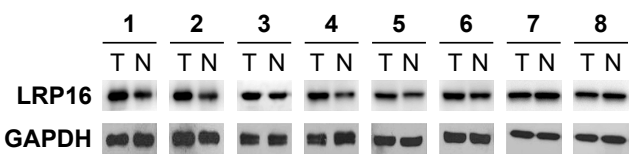

B

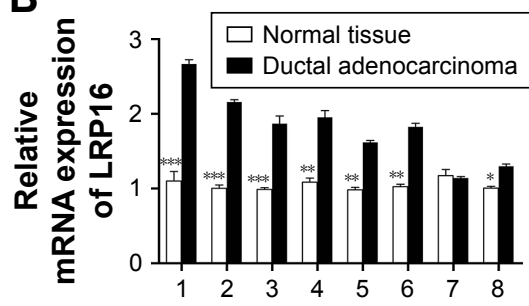

C

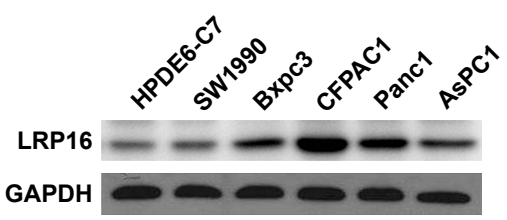

E

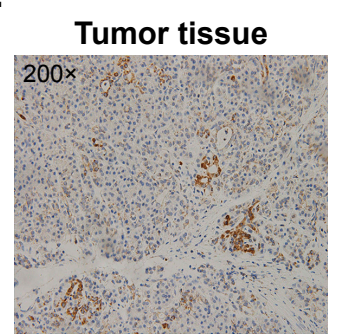

D

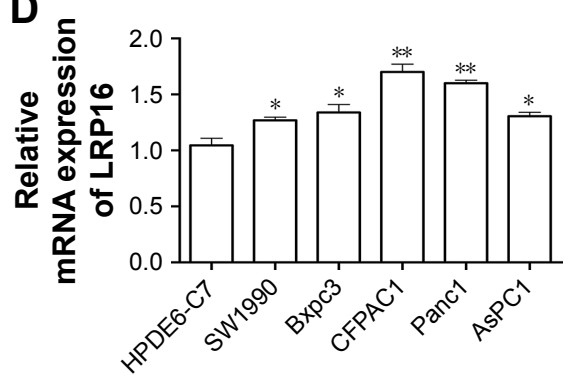

Adjacent nontumor

tissue

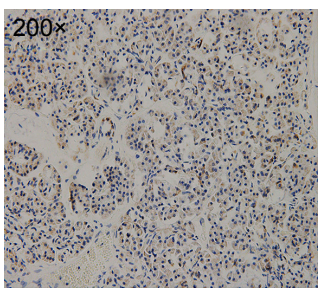

Figure I LRPI6 expression in pancreatic cancer cell lines and surgical specimens.

Notes: (A) The expressions of LRPI 6 in eight pairs of pancreatic cancer tissues and normal pancreatic tissues were evaluated by Western blotting ( $\mathrm{N}=$ normal liver tissues, $T=$ tumor tissues). (B) The mRNA levels of LRPI6 expression in eight pairs of pancreatic cancer tissues and normal pancreatic tissues were detected by qPCR. Data shown represent mean \pm SD for triplicate samples from a representative experiment. $* P<0.05 ; * * P<0.01$; $* * * P<0.00$ I. (C and D) Normal pancreatic cell line HPDE6-C7 and five pancreatic cancer cell lines were subjected to Western blotting and qPCR to determine the intracellular LRPI6 expression, and GAPDH was used as loading control. (E) Immunohistochemistry for LRPI6 expression in pancreatic cancer samples and nontumor samples (200×).

of which the LRP16 protein expression was noted in cell nucleus or/and cytoplasm, while the nontumor tissues were regarded as LRP16 negative (Figure 1E).

\section{The relationship between LRPI6 expression and clinical parameters and prognosis}

Then, we further determined the relationship between LRP16 expression and clinical significance. IHC analysis showed that the frequency of positive staining was $60.9 \%$ for LRP16 and in the paraffin sections $(n=156,2005-2010)$ stored in our hospital as mentioned Tissue specimens and patients (Table 1). The expression of LRP16 was associated with gender of the patients $(P=0.04)$, tumor size $(P<0.01)$, tumor location $(P<0.01)$, clinical stage $(P<0.01)$, distant metastasis $(P<0.01)$, and tumor differentiation. However, there was no association between LRP16 expression and the ages of patients $(P=0.3)$ or perineural invasion $(P=0.068)$ (Table 1$)$.

To evaluate the effect of LRP16 expression on survival, patients were classified as LRP16 positive and
LRP16 negative. The median OS was 23 months (range 5-54.5 months). The evaluation for OS by Kaplan-Meier method demonstrated that patients with positive LRP16 expression had poorer prognosis (Figure 2). PC patients with positive LRP16 expression had a median OS of 19 months and that of the LRP16 negative PC patients was 33 months. There was statistical difference between the groups $(P<0.001$, Figure 2$)$.

The univariate analysis was conducted and the prognostic factors with a $P$-value of $<0.05$ were included into multivariate Cox regression analysis. The results demonstrated that tumor size $(P=0.001)$, the presence of perineural invasion $(P=0.02)$, clinical stage $(P<0.001)$, distant metastasis $(P=0.015)$, and LRP16 expression $(P<0.001)$ were prognostic factors with statistical significance in univariate analysis (Table 2), whereas age $(P=0.617)$, gender $(P=0.429)$, and tumor differentiation $(P=0.313)$ showed no prognostic value. Through multivariate analysis, we found that tumor size $(P=0.019)$, clinical stage $(P=0.029)$, and LRP16 expression $(P=0.011)$ were independent prognostic factors for $\mathrm{PC}$; 


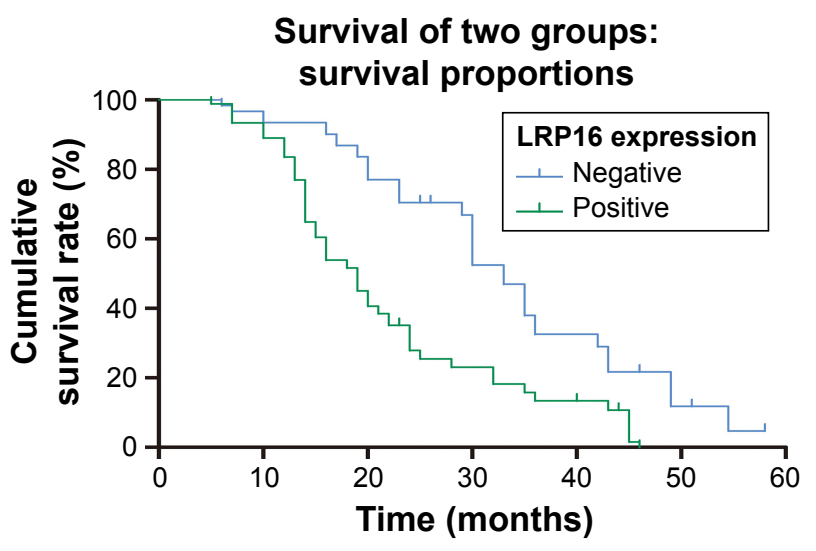

Figure 2 Clinical significance of LRPI6 expression in the prognosis of pancreatic cancer.

Note: Cumulative survival rate of PC patients with positive and negative LRPI6 expressions $(P<0.001)$.

Abbreviation: PC, pancreatic carcinoma.

however, perineural invasion $(P=0.247)$ and distant metastasis $(P=0.193)$ lost the prognostic value (Table 2$)$.

\section{LRPI6 promotes cell proliferation, migration, and invasion in PC cell lines}

Our data mentioned earlier suggests that the increased expression of LRP16 in PC specimens correlated with poor outcome in PC patients. Therefore, we further evaluated the roles of LRP16 in the cell proliferation, migration, and invasion ability in PC cell lines. LRP16 protein was highly expressed in Panc1 and CFPAC1 cell lines (Figure 1C and D). Specific shRNA for LRP16 was stably transfected into Panc1 and CFPAC1 cells to silence LRP16 expression. The efficiency of LRP16 knockdown was confirmed through qRT-PCR and Western blotting analysis, the LRP16 level was barely detected after transfection
(Figure 3A). Then, we determined the proliferation, apoptosis, and tumorigenic properties of PC cell lines upon LRP16 knockdown. As shown in Figure 3B and C, reduced LRP16 expression markedly decreased tumor growth but increased apoptosis both in Panc1 and CFPAC1 cells. Moreover, knockdown of LRP16 reduced cell migration and invasion based on observations from transwell assays (Figure 3D).

Furthermore, we used lentiviral transduction system to overexpress LRP16 in PC cell lines SW1990 and AsPC1 which has been proved to have lower endogenous LRP16 expression (Figure 1C and D). qRT-PCR and Western blotting analyses verified the overexpression efficiency (Figure 4A). The results showed that compared with control, LRP16 overexpression increased cell proliferation and promoted cell migration and invasion but inhibited apoptosis $(P<0.05)$ (Figure 4B-D).

\section{LRPI6 expression promotes tumor growth in the NOD/SCID mouse model}

Based on the above findings, we further performed tumor formation experiments in xenograft models using NOD/ SCID mice to assess the effect of LRP16 on tumor growth in vivo. Briefly, LRP16-knockdown Panc1 cells and their control cells (Panc1 cells) were grown as xenograft tumors in NOD/SCID mice through subcutaneous injection and tumor volumes and survival time were then measured. As shown in Figure 5A and B, the silence of LRP16 significantly decreased tumor growth and prolonged the survival compared with control group. Moreover, we tested how LRP16 overexpression affected tumor growth and survival time in vivo with Panc1 cell line. The tumor volume

Table 2 The univariate and multivariate analyses of the prognostic factors

\begin{tabular}{|c|c|c|c|c|c|c|}
\hline \multirow[t]{2}{*}{ Factors } & \multicolumn{3}{|c|}{ Univariate analysis } & \multicolumn{3}{|c|}{ Multivariate analysis } \\
\hline & HR & $95 \% \mathrm{Cl}$ & $P$-value & HR & $95 \% \mathrm{Cl}$ & $P$-value \\
\hline \multicolumn{7}{|l|}{ Age (years) } \\
\hline$\geq 65$ vs $<65$ & 1.042 & $0.672-1.329$ & 0.617 & & & \\
\hline \multicolumn{7}{|l|}{ Gender } \\
\hline Male vs female & 0.945 & $0.583-1.242$ & 0.429 & & & \\
\hline Tumor size $(\mathrm{cm})$ & & & & & & 0.019 \\
\hline$>4$ vs $\leq 4$ & 2.406 & $1.579-3.938$ & 0.001 & 2.077 & $1.354-3.176$ & \\
\hline Perineural invasion & & & & & & 0.247 \\
\hline Present vs absent & 3.172 & $1.966-5.319$ & 0.02 & 1.89 & $0.754-2.763$ & \\
\hline Clinical stage & & & & & & 0.029 \\
\hline Advanced stage vs early stage & 2.676 & $1.431-3.599$ & $<0.001$ & 1.917 & $1.368-2.725$ & \\
\hline Distant metastasis & & & & & & 0.193 \\
\hline Yes vs no & 1.701 & $1.288-3.574$ & 0.015 & 1.179 & $0.885-2.719$ & \\
\hline \multicolumn{7}{|l|}{ Differentiation } \\
\hline Well vs moderate vs poor & 1.136 & $0.659-1.946$ & 0.313 & & & \\
\hline LRPI6 expression & & & & & & 0.011 \\
\hline Positive vs negative & 2.725 & $1.403-3.879$ & $<0.001$ & 1.635 & $1.29 \mid-2.504$ & \\
\hline
\end{tabular}


A
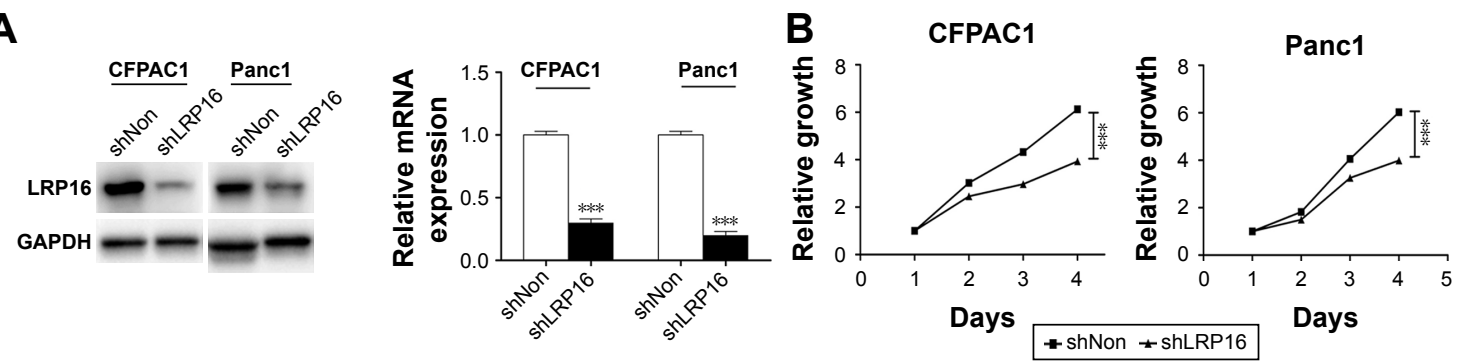

C
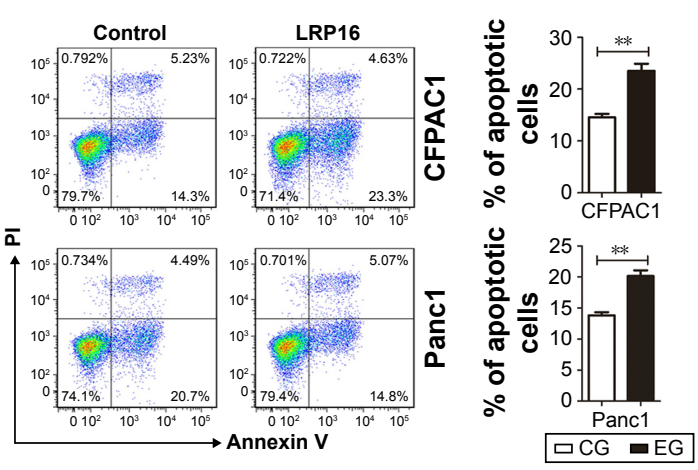

D

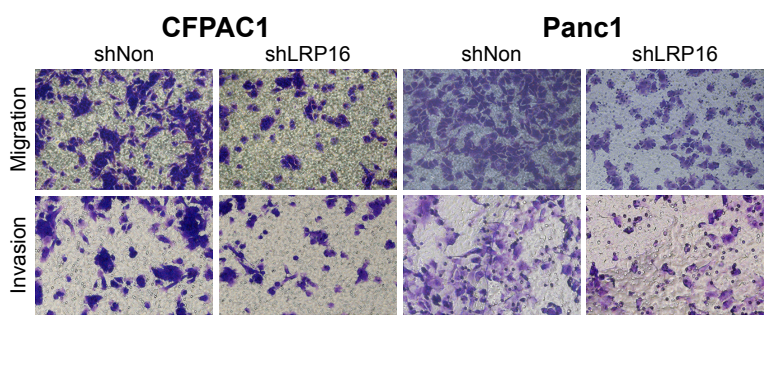

Figure 3 The effects of LRPI6 knock-down on pancreatic cancer cell proliferation, apoptosis, and migration.

Notes: (A) Knock-down of LRPI6 was evaluated by qPCR and Western blotting in CFPACI and Pancl cells. (B) The proliferation of CFPACI and Pancl cells was assessed by the MTS assay. Cells were seeded in triplicates into 96 -well plates for MTS assay at 24, 48, 72, and 96 hours. (C) The effect of knockdown LRPI6 on the apoptosis of pancreatic cancer cell was determined by flow cytometry. Cells were cultured in medium with no FBS for 48 hours and then stained with Pl and Annexin $\mathrm{V}$ for flow cytometric analysis. (D) The migration and invasion ability of CFPACI and Pancl cells were determined by transwell assay. The migrated and invasion cells were stained by crystal violet. $* * P<0.0$ I; $* * * P<0.00$ I.

Abbreviations: MTS, 3-(4,5-dimethyl-thiazol-2yl)-5-(3-carboxymethoxyphenyl)-2-(4-sulfophenyl)-2H-tetrazolium; CG, control group; EG, experiment group; FBS, fatal bovine serun; PI, Propidium lodide.

A

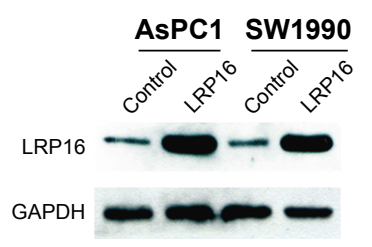

AsPC1

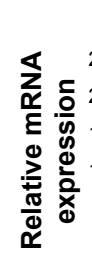

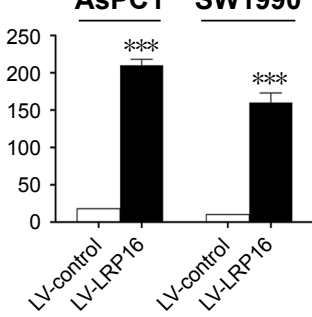

B

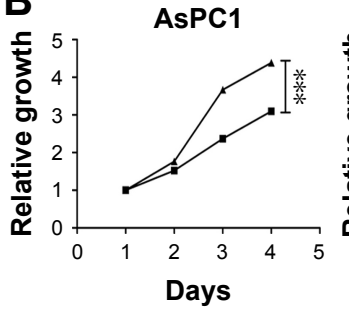

SW1990

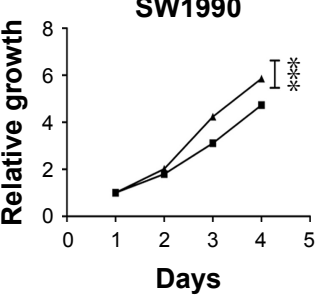

$\rightarrow$ LV-control + LV-LRP16
C
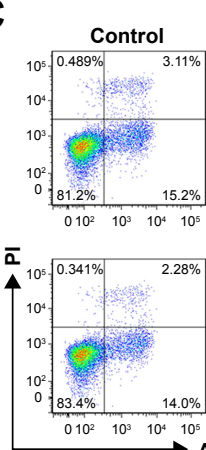
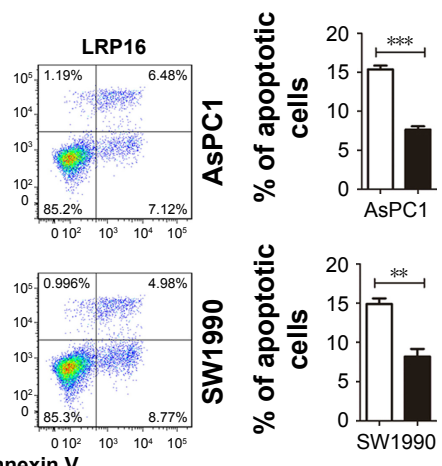

D
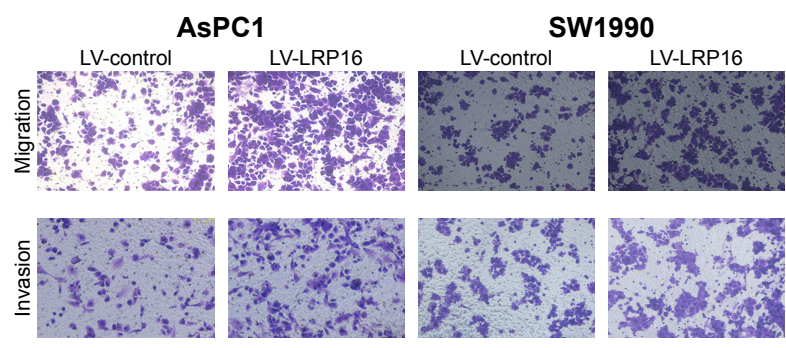

$\square \mathrm{CG} \square \mathrm{EG}$

Figure 4 The effects of overexpression of LRPI6 on pancreatic cancer cell proliferation, apoptosis, and migration.

Notes: (A) Overexpression of LRPI6 was verified by qPCR and Western blotting in AsPCI and SWI990 cells. (B) The proliferation of LRPI6-overexpression AsPCI and SWI 990 was measured by MTS assay. (C) The apoptosis of AsPCI and SW 1990 cell lines was determined by flow cytometry. (D) The migration ability of AsPCI and SWI990 with LRPI 6 overexpression was detected by transwell assay. $* * P<0.01 ; * * * P<0.001$.

Abbreviations: MTS, 3-(4,5-dimethyl-thiazol-2yl)-5-(3-carboxymethoxyphenyl)-2-(4-sulfophenyl)-2H-tetrazolium; LV, lentiviral vectors. 
A

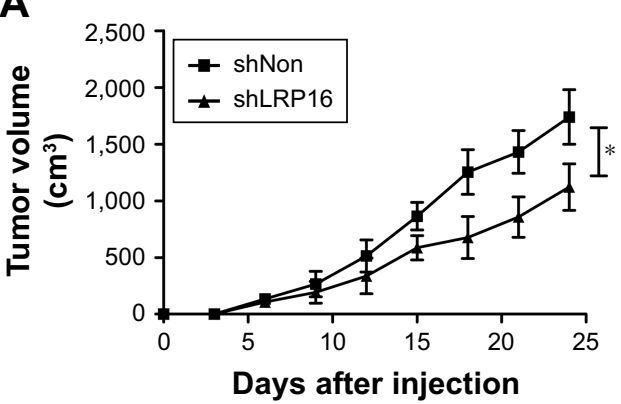

C

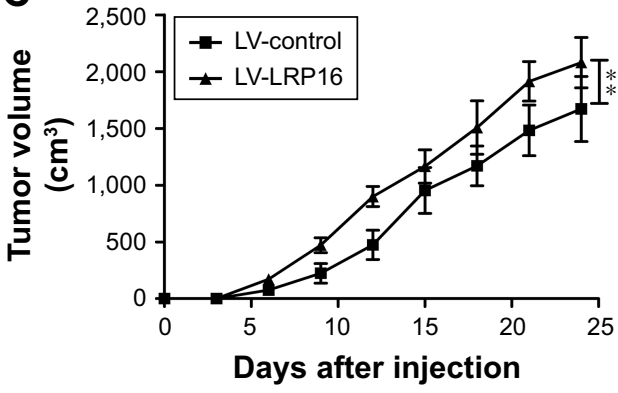

B
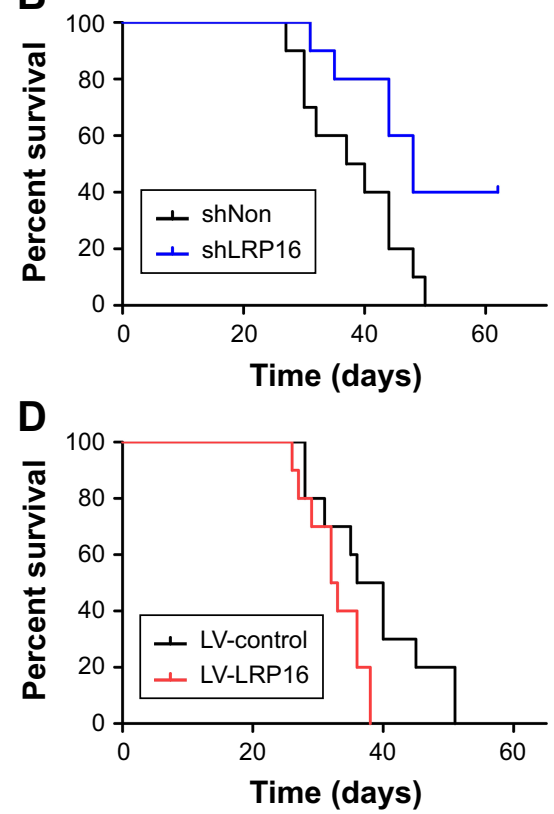

Figure 5 The effect of knockdown or overexpression of LRPI6 on the tumorigenesis of pancreatic cancer cells in vivo.

Notes: (A) Tumor growth of the mice injected with LRPI6-knockdown Pancl cells was measured every 3 days. (B) Survival curve of LRPI6 knockdown tumor bearing mice. (C and D) Tumor growth and survival curve of the mice injected with LRPI6-overexpression Pancl cells. ${ }^{*} P<0.05$; $* * P<0.0$ I.

Abbreviation: LV, lentiviral vectors.

of LRP16-overexpression xenografts was significantly increased compared with that of tumors formed by normal cells (Figure 5C). The overexpression of LRP16 shortened the survival of tumor-bearing mice (Figure 5D). Overall, our results indicate that LRP16 has oncogenic activity both in vitro and in vivo in PC.

\section{Discussion}

$\mathrm{PC}$ is a common and lethal malignancy in digestive system. Although numerous individual and cooperative studies have made contributions to the prevention and treatment of PC, the survival rate remains low. ${ }^{11}$ Predictive and prognostic factors are needed to stratify high-risk patients and provide implications for treatment options. We saw positive expression of LRP16 in tumors relative to normal tissues from surgical specimens of PC patients, suggesting a role for this gene in human tumorigenesis. Our results further show that knockdown of LRP16 results in decreased PC growth in vitro and in vivo. Deletion of LRP16 in xenograft models using NOD/SCID mice of PC was sufficient to decrease tumor growth and improve OS, suggesting that direct targeting of LRP16 would be beneficial in PDAC.

Our data also show that positive expression of LRP16 in tumors is associated with gender, tumor size, tumor location, clinical stage, distant metastasis, and tumor differentiation in PC patients undergoing curative surgery. The survival analysis showed that patients with positive
LRP16 expression had shorter OS, suggesting that LRP16 was an independent prognostic factor for PC patients. Associations between increased LRP16 expression and poor clinical outcomes have been observed in some other cancers; however, a direct link between LRP16 and migration or apoptosis in $\mathrm{PC}$ has not been shown. ${ }^{6,8}$ In the present study, we provide direct evidence that LRP16 is sufficient to promote metastasis in $\mathrm{PC}$, as its increased expression promotes migration and invasion, and its knockdown inhibits cancer cell transfer. As positive LRP16 expression has also been linked to poor patient outcomes, we suggest that direct targeting of LRP16 may improve patient outcomes by limiting tumor metastasis.

LRP16 was originally identified as a leukemia relapserelated gene. ${ }^{12}$ Further studies had showed that LRP16 gene also played pivotal roles in the development of hormonedependent breast cancer. ${ }^{13,14}$ Recent studies showed that LRP16 has been found expressed at a high level in gastric carcinoma, ovarian carcinoma, and colorectal carcinoma. ${ }^{6,8,15}$ More recent work has revealed that LRP16 took part in the activation of NF- $\kappa B$, which was an important event for the modulation of the response to DNA double-strand breaks. ${ }^{16}$ Moreover, LRP16 was not only a crucial regulator for $\mathrm{NF}-\kappa \mathrm{B}$ activation inside the nucleus but also pivotal stimuli to the aberrant activation of NF- $\mathrm{KB}$ in tumors. ${ }^{17}$ These findings suggest that LRP16 contributes to the development of carcinogenesis in various tumors. In the present study, 
we found that the expression of LRP16 increased significantly in PDAC tissues and analyzed the relationship between LRP16 expression and clinical significance. We also provided in vitro and in vivo evidence to support that the expression levels of LRP16 positively correlated with proliferation, migration, and invasion in $\mathrm{PC}$.

\section{Conclusion}

The present study supports a requirement for LRP16 in tumorigenesis, migration, and invasion of PC cell lines that helps explain the associated findings of positive LRP16 expression in PC tumors and the relative poor clinical outcomes. Our data suggest that the targeting of LRP16 may be a promising approach for treating PC and the detection of LRP16 expression with immunohistochemistry may serve as an independent and useful biomarker to predict the prognosis in PC patients. Due to the limitations in this study, more large-scale studies are warranted. Moreover, the molecular mechanism of the signaling pathways accounting for the associations between LRP16 and clinical features of PC need to be clarified in further studies.

\section{Acknowledgments}

This work was supported by grants from the Ministry of Science \& Technology ("973" projects) (numbers 2012CB917104 and 2014CB541805) and the National Natural Science Foundation of China (numbers 81172853 and 81341069).

\section{Disclosure}

The authors report no conflicts of interest in this work.

\section{References}

1. Torre LA, Bray F, Siegel RL, Ferlay J, Lortet-Tieulent J, Jemal A. Global cancer statistics, 2012. CA Cancer J Clin. 2015;65(2):87-108.
2. Siegel RL, Miller KD, Jemal A. Cancer statistics, 2015. CA Cancer J Clin. 2015;65(1):5-29.

3. Khare V, Alam N, Saneja A, Dubey RD, Gupta PN. Targeted drug delivery systems for pancreatic cancer. J Biomed Nanotechnol. 2014;10(12): 3462-3482.

4. Vincent A, Herman J, Schulick R, Hruban RH, Goggins M. Pancreatic cancer. Lancet. 2011;378(9791):607-620.

5. Han WD, Yu L, Lou FD, et al. The application of RACE technique to clone the full-length cDNA of a novel leukemia associated gene LRP16. Zhongguo Shi Yan Xue Ye Xue Za Zhi. 2001;9(1):18-21.

6. Xi HQ, Zhao P, Han WD. Clinicopathological significance and prognostic value of LRP16 expression in colorectal carcinoma. World $J$ Gastroenterol. 2010;16(13):1644-1648.

7. Shao Y, Li X, Lu Y, Liu L, Zhao P. Aberrant LRP16 protein expression in primary neuroendocrine lung tumors. Int J Clin Exp Pathol. 2015; 8(6):6560-6565.

8. Li YZ, Zhao P, Han WD. Clinicopathological significance of LRP16 protein in 336 gastric carcinoma patients. World J Gastroenterol. 2009; 15(38):4833-4837.

9. Liao DX, Han WD, Zhao YL, et al. Expression and clinical significance of LRP16 gene in human breast cancer. Ai Zheng. 2006;25(7):866-870.

10. Zhao $\mathrm{P}, \mathrm{Lu} \mathrm{Y}, \mathrm{Han} \mathrm{W}$. Clinicopathological significance and prognostic value of leukemia-related protein 16 expression in invasive Ductal breast carcinoma. Cancer Sci. 2010;101(10):2262-2268.

11. Feig C, Gopinathan A, Neesse A, Chan DS, Cook N, Tuveson DA. The pancreas cancer microenvironment. Clin Cancer Res. 2012;18(16): 4266-4276.

12. Zhao YL, Han WD, Li Q, et al. Mechanism of transcriptional regulation of LRP16 gene expression by 17-beta estradiol in MCF-7 human breast cancer cells. J Mol Endocrinol. 2005;34(1):77-89.

13. Han WD, Zhao YL, Meng YG, et al. Estrogenically regulated LRP16 interacts with estrogen receptor alpha and enhances the receptor's transcriptional activity. Endocr Relat Cancer. 2007;14(3):741-753.

14. Han WD, Mu YM, Lu XC, et al. Up-regulation of LRP16 mRNA by 17beta-estradiol through activation of estrogen receptor alpha (ERalpha), but not ERbeta, and promotion of human breast cancer MCF-7 cell proliferation: a preliminary report. Endocr Relat Cancer. 2003;10(2): 217-224.

15. Tian L, Wu Z, Zhao Y, et al. Differential induction of LRP16 by liganded and unliganded estrogen receptor alpha in SKOV3 ovarian carcinoma cells. J Endocrinol. 2009;202(1):167-177.

16. Wu Z, Wang C, Bai M, et al. An LRP16-containing preassembly complex contributes to NF-kappaB activation induced by DNA doublestrand breaks. Nucleic Acids Res. 2015;43(6):3167-3179.

17. Wu Z, Li Y, Li X, et al. LRP16 integrates into NF-kappaB transcriptional complex and is required for its functional activation. PLoS One. 2011;6(3):e18157.
OncoTargets and Therapy

\section{Publish your work in this journal}

OncoTargets and Therapy is an international, peer-reviewed, open access journal focusing on the pathological basis of all cancers, potential targets for therapy and treatment protocols employed to improve the management of cancer patients. The journal also focuses on the impact of management programs and new therapeutic agents and protocols on
Dovepress

patient perspectives such as quality of life, adherence and satisfaction. The manuscript management system is completely online and includes a very quick and fair peer-review system, which is all easy to use. Visit http://www.dovepress.com/testimonials.php to read real quotes from published authors. 